\title{
Resilience, occupational burnout, and parenting stress in nurses caring for COVID-19 patients
}

\author{
Fateme Mohammadi ${ }^{1}$ Mostafa Bijani², Seyed Reza Borzou ${ }^{3}$ Khodayar Oshvandi ${ }^{4}$, Salman Khazaei ${ }^{5}$, \\ Saeid Bashirian ${ }^{6}$ \\ ${ }^{1}$ Chronic Diseases (Home Care) Research Center and Autism Spectrum Disorders Research Center, Department of Nursing, \\ Hamadan University of Medical Sciences, Hamadan, Iran \\ ${ }^{2}$ Department of Medical Surgical Nursing, Fasa University of Medical Sciences, Fasa, Iran \\ ${ }^{3}$ Department of Medical Surgical Nursing, School of Nursing and Midwifery, Chronic Diseases (Homecare) Research Center, \\ Hamadan University of Medical Sciences, Hamadan, Iran \\ ${ }^{4}$ Department of Medical Surgical Nursing, School of Nursing and Midwifery, Mother and Child Care Research Center, \\ Hamadan University of Medical Sciences, Hamadan, Iran \\ ${ }^{5}$ Health Sciences Research Center, Health Sciences \& Technology Research Institute, Hamadan University of Medical \\ Sciences, Hamadan, Iran \\ ${ }^{6}$ Department of Public Health, School of Health, Social Determinants of Health Research Center, Health Sciences \\ \& Technology Research Institute, Hamadan University of Medical Sciences, Hamadan, Iran
}

Neuropsychiatria i Neuropsychologia 2021; 16, 3-4: 116-123

Address for correspondence:

Saeid Bashirian, PhD

Assoc. Prof. of Health Education and Promotion

Department of Public Health

School of Health

Social Determinants of Health Research Center,

Health Sciences \& Technology Research Institute,

Hamadan University of Medical Sciences

Hamadan, Iran

e-mail: s-bashirian@umsha.ac.ir

\section{Abstract}

Introduction: Care of patients with coronavirus disease may have an impact on the occupational burnout, resilience, and parenting of nurses. The study was performed to evaluate occupational burnout, resilience, and parenting stress in nurses caring for COVID-19 patients.

Material and methods: This cross-sectional study was based on Strengthening the Reporting of Observational Studies in Epidemiology (STROBE) statement. A total of 630 nurses caring for COVID-19 patients in 5 hospitals were selected via convenience sampling. Participants completed the scales online. Data were analyzed in SPSS v. 22. Results: A total of 420 nurses completed and returned the questionnaires. The resilience mean score of the nurses who participated in the present study was $32.33 \pm 2.57$ and the occupational burnout mean score was 32.33 \pm 2.57 . Also, the parenting stress mean score of the 310 nurses who were married and had children was found to be $17.53 \pm 1.58$ during the COVID-19 crisis. Occupational burnout has a negative correlation with resilience and a positive correlation with parenting stress and can predict $61.32 \%$ of changes in the occupational burnout variance of nurses

Conclusions: Resilience, parenting stress, marital status, number of children, employment status, and gender predicted a high percentage of the nurses' occupational burnout variance. The nurse managers should use these findings to provide appropriate environments for nurses, to develop more comprehensive plans in support of nurses for the current and future crises.

Key words: occupational burnout, resilience, parenting stress, nurses, coronavirus, COVID-19.

\section{Introduction}

Coronavirus disease 2019 (COVID-19) emerged in China in 2019, very quickly became a pandemic, and has caused a large number of deaths in many countries so far (WHO 2020). The initial symptoms of COVID-19 are similar to those of influenza, but the infection gradually develops and causes severe cardiopulmonary and renal disorders, and can eventually cause death (Wu and McGoogan 2020). As a little-known disease, its genetic structure constantly changes, new symptoms are continuously recorded in the infected people, and, most importantly, there is not a definite cure or specialized vaccine for it. Thus, over 8.3 million people in the world 
have contracted the disease in the past three months, over 224,000 of whom have lost their lives as a result (Novel Coronavirus 2019; Lai et al. 2020a).

In Iran, however, the spread of COVID-19 has been complex. At the beginning of the pandemic, Iran had the third highest reported number of cases of COVID-19, after China and Italy. Currently, Iran is faced with the third wave of the pandemic, while many other countries are going through the second wave (Mohammadi et al. 2021; Mohammadi et al. 2020). Although the majority of Iranians wear masks, the inappropriate economic situation has resulted in few restrictions on work, social activity and travel in all cities of Iran. As a result, large numbers of people use public transportation daily, streets are crowded, all employees are at work, and telecommuting from home is not common (Nemati et al. 2020). These factors have caused about 530,500 cases of infection, over 30,500 of which led to death of the patients (Nemati et al. 2020).

The high worldwide fatality rate of COVID-19 has caused many people to experience considerable tension, with adverse effects on their social activities and psychological security (Liu et al. 2020). Due to the special nature of their occupation, nurses are at higher risk of suffering serious physical and psychological harm, including stress and depression. It is obvious that these psychological crises can influence nurses' personal and family responsibilities as well, subject them to occupational burnout, and adversely affect parental duties (Greenberg et al. 2020; Lai et al. 2020b). In January 2020, the World Health Organization (WHO) declared the COVID-19 pandemic as a public health emergency of international concern (PHEIC) (Wang et al. 2020). Accordingly, nurses play a significant role in healthcare and treatment systems in caring for and improving the health of patients infected with the coronavirus (Greenberg et al. 2020; WHO 2020). However, caring for COVID-19 patients has subjected nurses to great psychological tension and work stress, hence the need for taking measures to protect nurses' psychological and physical well-being (WHO 2020). In this regard, Stuijfzand et al. (2020) stated that healthcare professionals during epidemics and pandemics (severe acute respiratory syndrome [SARS], Middle East respiratory syndrome coronavirus [MERS], Ebola virus disease) experience myriad mental health problems including psychological distress, insomnia, alcohol/drug misuse, posttraumatic stress disorder (PTSD), depression, anxiety, anger and burnout (Stuijfzand et al.
2020). Also, Lehmann et al. (2015) found that health care professionals reported depression, stress, social isolation, and job fatigue during the Ebola outbreak. Social isolation has been largely due to parenting stress and concerns about the transmission of the disease to children and other family members. On the other hand, although job stress and fatigue can affect tolerance, they have overcome these tensions and work pressures with team working and observing the principles of infection control (Lehmann et al. 2015). A study conducted by Liu et al. (2020) confirmed that, compared to other members of medical staff, nurses experience higher levels of anxiety and depression in caring for COVID-19 patients and face more challenges in professional duties, family responsibilities and daily activities.

The study of Roy et al. (2020) showed that nurses experience high degrees of stress, anxiety, depression and PTSD during the COVID-19 crisis. Thus, it is essential that the executive administrators of healthcare systems and the crisis committees of hospitals consider the status of nurses' psychological well-being, anxiety, depression, and occupational burnout. The present study is an attempt to measure occupational burnout, resilience, and parenting stress in nurses who care for COVID-19 patients in Iran.

\section{Material and methods}

\section{Study design and aims}

This cross-sectional study was conducted based on the Strengthening the Reporting of Observational Studies in Epidemiology (STROBE) statement from February to June 2020. We tried to achieve the following two aims: evaluation of occupational burnout, resilience, and parenting stress in nurses who care for COVID-19 patients; and investigating the relationship of occupational burnout with resilience, parenting stress and demographic characteristics in nurses who care for COVID-19 patients.

\section{Participants and sampling}

In this study, the sample size was estimated at 630 subjects according to the study of Roy et al., with $\beta=80 \%, \alpha=0.05$, and taking into account $10 \%$ attrition in each group. The participants were selected via convenience sampling. The correspondence author collected the emails of all the nurses working in COVID-19 wards from the office of nursing services, in 5 hospitals affiliated with the University of Medical Sciences in the west of Iran. Therefore, nurses 
who provided care to COVID-19 patients were invited via email and selected through convenience sampling to participate in the study. The inclusion criteria were the following: being willing to participate, being in practice in one of the hospitals assigned for COVID-19 patients, and having at least one month of work experience in caregiving wards for patients with COVID-19. The subjects who failed to answer more than half of the items on the questionnaires or did not turn in the questionnaires were excluded. The subjects were asked to complete and submit the questionnaires - a personal (demographic) characteristics questionnaire, an occupational burnout scale, a resilience scale, and a parenting stress scale (if they were parents) online. The researchers sent emails and reminder messages to the participants, so that the majority of the questionnaires (90\%) were gathered in June.

\section{Measurements}

\section{The occupational burnout scale}

Developed by Maslach in 1996, the Maslach Burnout Inventory is the most commonly used scale for measuring occupational burnout (Maslach et al. 1986). The scale consists of 22 items which address three dimensions of occupational burnout: 9 items for emotional exhaustion, 5 items for depersonalization, and 8 items for personal accomplishment. The items measure the frequency and degree of burnout on an 8-point Likert scale: from 0 (very little) to 7 (very high). In the domain of emotional exhaustion, scores of 27 and above indicate severe emotional exhaustion, scores of 16 and below show slight emotional exhaustion, and scores of between 17 and 26 denote average emotional exhaustion. In the domain of depersonalization, scores of above 13 represent severe depersonalization and in the domain of personal accomplishment, scores of below 31 reveal low levels of personal accomplishment. The internal reliability of this scale was calculated as a Cronbach's $\alpha$ of 0.80 (Maslach et al. 1986). In their study, Baher et al. find the Cronbach's $\alpha$ of the scale to be 0.79 (Baher 2009).

\section{The self-report Caregiver Burden Inventory}

The Caregiver Burden Inventory was developed by Novak and Guest in Canada in 1988 to measure caregivers' burden. The scale consists of 24 items which assess caregivers' burden in five domains: time-dependence burden ( 5 items: $1,2,3,4,5)$, developmental burden (5 items:
$6,7,8,9,10)$, physical burden (4 items: 11 , $12,13,14)$, social burden ( 5 items: 15,16 , $17,18,19)$, and emotional burden ( 5 items: $20,21,22,23,24)$. Completion of the scale requires approximately 15 minutes. The items are scored on a 5-point Likert scale ranging from 0 (Not at all) to 4 (Absolutely). The score range is between 0 and 96: scores of 0 to 32 indicate low burden, 33 to 64 moderate burden, and 65 to 96 great burden (Novak and Guest 1989). In their study conducted in Iran, Shafiezadeh et al. (2020) determined the reliability of the inventory at 0.82 , which is a satisfactory value.

\section{Parenting Stress Index-Short Form (PSI-SF)}

Created by Abidin in 1995, the Parenting Stress Index comprises 3 subscales: parental distress (items 1 to 12), parent-child dysfunctional interactions (items 13 to 24), and child characteristics (items 25 to 36). The scale measures parental stress on a 5-point Likert scale ranging from 1 (completely disagree) to 5 (completely agree) (Abidin et al. 2006). In their studies, Yeh et al. (2001) and Reitman et al. (2002) report the reliability of the scale to be 0.89 and 0.90 respectively. Assessing the reliability and validity of the scale in Iran, Shirzadi found the reliability of the various dimensions of the index to be between 0.59 and 0.86 , which are acceptable values (Shirzadi et al. 2015).

\section{Statistical methods}

After data collection, they were analyzed using descriptive statistics (frequency, percentage, mean, and standard deviation) in SPSS v. 22. To investigate the relationship between occupational burnout on the one hand and resilience and parenting stress on the other, the researchers applied the $\chi^{2}$ test, independent $t$-test, and ANOVA. The significance level was set at 0.05 . Subsequently, the variables of demographics, resilience, and parenting stress which were found to correlate with occupational burnout $(p<0.25)$ were entered into multiple linear regression with the backward technique. Before running the analysis of multiple linear regressions, the researchers examined the assumptions of normality of data, homogeneity of variance, and independence of residuals.

\section{Ethics approval and consent to participate}

The institutional review board of the medical universities located in the west of Iran provided ethics approval (approval number: 9904242498). 
Also at the beginning of study, the researcher got the emails of all the nurses working in COVID-19 wards from the office of nursing services, and invited them to participate in the study. In addition, the researcher introduced herself and explained the goals of the study and assured participants that all information would remain confidential and that they could withdraw from the study at any time. Finally, participants completed the written consent forms and sent them to the researcher by e-mail.

\section{Results}

A total of 420 subjects completed and returned the questionnaires via e-mail or a social network application, giving a response rate of $66.7 \%$. The nurses' reasons for not participating in this study were heavy workload, intensive shifts, and infection with COVID-19.

Of the 420 nurses who participated in the study, $71.4 \%$ were female and $28.6 \%$ were male. The range of the participants' ages was between 23 and 55 years with the mean of 35.24 \pm 3.56 years. The majority of the participants (73.8\%) were married, $54.8 \%$ of whom had two children. Also, most of the participants had a bachelor of science degree in nursing $(63.1 \%)$, were contractual employees (50.5\%), had 11 years of work experience, and worked 32 rotating shifts per month (Table 1). The findings of the study showed that there was a statistically significant relationship between occupational burnout on the one hand and marital status, number of children, employment status, and gender on the other.

\section{Resilience, occupational burnout and parenting stress in the participants}

The resilience mean score of the nurses who participated in the present study was 32.33 \pm 2.57 and the occupational burnout mean score was $32.33 \pm 2.57$. Also, the parenting stress mean score of the 310 nurses who were married and had children was found to be 17.53 \pm 1.58 during the COVID-19 crisis (Table 2).

\section{Relationship between participants' resilience, occupational burnout, and parenting stress}

The findings of the study show that there is a strong and inverse correlation between occupational burnout and resilience in nurses who care for COVID-19 patients $(p<0.001$, $r=-0.70)$. Also, a stronger and more inverse correlation was found to exist between occupa-

Table 1. Participants' demographic characteristics and occupational burnout scores

\begin{tabular}{|c|c|c|c|c|}
\hline \multicolumn{2}{|c|}{ Demographic variables } & \multirow{2}{*}{$\begin{array}{l}\text { Number (\%) } \\
197 \text { (46.9) }\end{array}$} & \multirow{2}{*}{$\begin{array}{l}\text { Occupational burnout } \\
\text { Mean } \pm \text { SD } \\
38 \pm 1.21\end{array}$} & \multirow{2}{*}{$\begin{array}{c}p \text {-value } \\
0.324\end{array}$} \\
\hline Age (years) & $23-33$ & & & \\
\hline & $34-44$ & $154(36.7)$ & $37 \pm 1.96$ & \\
\hline & $45-55$ & $69(16.4)$ & $37 \pm 1.43$ & \\
\hline \multirow[t]{2}{*}{ Gender } & Female & $300(71.4)$ & $41 \pm 2.12$ & 0.042 \\
\hline & Male & $120(28.6)$ & $38 \pm 1.87$ & \\
\hline \multirow[t]{2}{*}{ Marital status } & Single & $110(26.2)$ & $32 \pm 2.76$ & 0.033 \\
\hline & Married & $310(73.8)$ & $38 \pm 1.54$ & \\
\hline \multirow[t]{4}{*}{ Number of children } & None & $12(3.9)$ & $35 \pm 2.32$ & 0.031 \\
\hline & 1 & $80(25.8)$ & $38 \pm 1.61$ & \\
\hline & 2 & $170(54.8)$ & $39 \pm 2.14$ & \\
\hline & More than 2 & 48 (15.5) & $42 \pm 1.87$ & \\
\hline \multirow[t]{3}{*}{ Education } & Associate & 73 (17.4) & $31 \pm 1.32$ & 0.451 \\
\hline & Bachelor & $265(63.1)$ & $32 \pm 1.05$ & \\
\hline & Master & 82 (19.5) & $32 \pm 1.08$ & \\
\hline \multirow[t]{2}{*}{ Employment status } & Permanent & $208(49.5)$ & $33 \pm 2.69$ & 0.039 \\
\hline & Contractual & $212(50.5)$ & $38 \pm 1.57$ & \\
\hline \multirow[t]{3}{*}{ Work experience } & $\begin{array}{l}\text { Less than } \\
10 \text { years }\end{array}$ & $150(35.7)$ & $36 \pm 2.96$ & 0.310 \\
\hline & 10-20 years & $197(46.9)$ & $37 \pm 1.52$ & \\
\hline & 20-30 years & 73 (17.4) & $37 \pm 2.24$ & \\
\hline
\end{tabular}


Table 2. Means and standard deviations of participants' resilience, occupational burnout and parenting stress scores

\begin{tabular}{llcc} 
Variable & \multicolumn{1}{c}{ Dimension } & Mean \pm SD \\
Resilience & Time-dependence burden & $\begin{array}{c}\text { Total } \\
\text { Mean } \pm \text { SD }\end{array}$ \\
& Developmental burden & $32.33 \pm 2.57$ \\
& Physical burden & $14.32 \pm 1.42$ & \\
\cline { 2 - 3 } & Social burden & $15.76 \pm 2.18$ & \\
\cline { 2 - 3 } & Emotional burden & $10.42 \pm 1.53$ & \multirow{2}{*}{$32.33 \pm 2.57$} \\
\hline Occupational burnout & Emotional exhaustion & $16.78 \pm 1.34$ & \\
\cline { 2 - 3 } & Depersonalization & $10.11 \pm 1.28$ & \\
\cline { 2 - 3 } & Personal accomplishment & $48 \pm 3.22$ & \\
\hline Parenting stress & Parental stress & $39 \pm 2.53$ & \\
\cline { 2 - 3 } & dysfunctional interactions & $18 \pm 1.09$ & $17 \pm 2.53$ \\
\cline { 2 - 3 } & Child characteristics & $15 \pm 1.12$ & \\
\hline
\end{tabular}

Table 3. Relationship between resilience, occupational burnout, and parenting stress in married and single nurses and all participants

\begin{tabular}{lll}
\hline \multirow{2}{*}{ Occupational burnout } & Resilience (married nurses) & $r=-0.71, p<0.001$ \\
\cline { 2 - 3 } & Resilience (single nurses) & $r=-0.69, p<0.001$ \\
\cline { 2 - 3 } & Resilience (total) & $r=-0.70, p<0.001$ \\
\hline Occupational burnout & Parenting stress (married nurses) & $r=0.74, p<0.001$ \\
\hline
\end{tabular}

tional burnout and resilience in married nurses than single nurses. The results showed a positive correlation between the occupational burnout and parenting stress scores of the nurses with children $(p<0.001, r=0.74)$ (Table 3).

Predictor variables of occupational burnout in nurses who care for COVID-19 patients

The variable of resilience, parenting stress, number of children, marital status, employment status, and gender which had a $p$-value of smaller than 0.25 were entered into multiple linear regressions with the backward technique. These variables remained in the model and accounted for about $61.32 \%$ of the occupational burnout variance in the nurses who provided care to COVID-19 patients (Table 4).

\section{Discussion}

This study showed that nurses have reported high levels of occupational burnout, low levels of resilience, and high levels of tension in their relationship with children. Although a few studies have addressed work stress, knowledge, and awareness of nurses who care for COVID-19 patients, there are not any studies of resilience, occupational burnout, or parenting stress in this group of caregivers. Therefore, the researchers had to use articles which measure resilience, occupational burnout, or parenting stress in nurses who care for patients with other specific diseases. The occupational burnout mean score of the nurses was found to be $32.33 \pm 2.57$, which indicates high burnout. Occupational burnout in nurses is an issue of concern in nurs-

Table 4. Predictor variables of occupational burnout in nurses who care for COVID-19 patients

\begin{tabular}{|c|c|c|c|c|c|}
\hline \multirow[t]{2}{*}{ Variable } & \multicolumn{2}{|c|}{ Unstandardized coefficients } & \multirow{2}{*}{$\frac{\text { Standardized coefficients }}{\beta}$} & \multirow[t]{2}{*}{$\mathrm{T}$} & \multirow[t]{2}{*}{$p$-value } \\
\hline & B & SD & & & \\
\hline Resilience & -0.634 & 2.53 & -0.643 & -3.32 & 0.001 \\
\hline Parenting stress & 0.568 & 2.49 & 0.572 & 2.78 & 0.001 \\
\hline Number of children & 0.113 & 2.28 & 0.441 & 2.54 & 0.012 \\
\hline Marital status & 0.298 & 2.54 & 0.301 & 3.87 & 0.024 \\
\hline Employment status & 0.382 & 2.34 & 0.214 & 1.98 & 0.022 \\
\hline Gender & 0.331 & 1.89 & 0.186 & 1.64 & 0.039 \\
\hline
\end{tabular}


ing associations. Studies show that the physical and psychological pressures of caring for patients lead to occupational burnout in the long run, and thus nurses' length of service, work shifts, and salary must be redefined according to the type of patients they care for and the units where they work (Salahian et al. 2012). However, most of these studies report the occupational burnout of nurses with 5 years or more of work experience to be average, which is not consistent with the findings of the present study (Salahian et al. 2012; Sandeep et al. 2019; Velando-Soriano et al. 2020). This discrepancy can be attributed to the sudden emergence of COVID-19, which is highly infectious and has significantly increased nurses' workload and caused them to have to work longer shifts and stay away from their families in the past few months. The results of the present study show a statistically significant difference between the married and single participants in terms of their occupational burnout scores $(p<0.033)$. This difference may be due to the fact that, in addition to stress in the workplace, married nurses, especially those with children, are subject to greater psychological tension $(p<0.031)$ as a result of disruption in marital and parental duties (Da Silva et al. 2019a; Da Silva Azevedo et al. 2019b; Salahian et al. 2012; Sofology et al. 2019). The results of the study also show that contractual nurses $(p<0.039)$ and female nurses $(p<0.041)$ suffer from greater occupational burnout. Job insecurity, lower salaries, and the lower physical-psychological resilience of female nurses can account for the statistically significant difference between the occupational burnout scores of contractual and permanent nurses and male and female nurses (Salahian et al. 2012; Sofology et al. 2019).

The study of Salahian et al. (2012) shows that nurses' employment status and gender have an impact on their occupational burnout. In the present study, the resilience mean score of the nurses was found to be $32.33 \pm 2.57$, which is considered low (Amini 2013; Edward and Hercelinskyj 2007). In contrast, other studies report average to high levels of resilience for nurses, even for those who provide care to critically or terminally ill patients. The discrepancy can be attributed to lack of a definite treatment for COVID-19 and the high speed and rate of the infection, which have subjected nurses to severe physical and psychological tension with extremely adverse effects on their resilience (Amini 2013; Edward and Hercelinskyj 2007; Torgheh and Alipour 2015). The parenting stress mean score of 298 participants was found to be 17.53 \pm 1.58 . There are studies on parenting stress in working mothers or parents with a chronically ill child (Anthony et al. 2005; Van der Geest et al. 2014); however, there has not been much research into parenting stress in nurses. This lack of research may be due to the fact that nurses who work in rotating shifts can spend at least a few hours a day with their children and attend upbringing. But in the COVID-19 crisis, many nurses have to work longer hours caring for the infected and, due to the high transmissibility of the disease, would rather reside in hospitals temporarily or have relatives take away their children to their own homes to ensure safety. Stress in the workplace, fear of transmitting the infection to children, having to stay away from children, and inability to monitor children's upbringing have subjected nurses with children to extra psychological tension and, consequently, elevated occupational burnout in them.

The results of the present study show a strong and inverse correlation between the participants' occupational burnout and resilience, and a strong and direct correlation between the participants' occupational burnout and parental stress. In addition, the variables of resilience, parenting stress, number of children, marital status, employment status, and gender explained about $61.32 \%$ of the occupational burnout variance in the nurses who provided care to COVID-19 patients. There has been no study on the evaluation and prediction of the relationship between occupational burnout and resilience, parenting stress and demographic characteristic in nurses at the same time. In this regard, Amini stated that there is an inverse relationship between occupational burnout and resilience of nurses (Amini 2013). Also, Zou et al. (2016) found that occupational burnout was negatively related to resilience, which is consistent with the results of the present study. In addition, Salahian $e t$ al. stated that nurses who worked in the infectious diseases ward reported more occupational burnout than nurses in other wards. Also, job stress, role clarity, and workload predicted about $36.5 \%$ of the nurses' occupational burnout variance (Salahian et al. 2012). However, nurses in the present study considered that rapid transmission of the disease and their high workload increased occupational burnout and consequently decreased resilience. Rafiee et al. (2007) and Ahmadi et al. (2014) found that individual characteristics of nurses (gender, work experience) and workplace affect nurses' occupational burnout, which is consistent with the present study. 
Finally, the results of the study show that nurses who provide care to COVID-19 patients and have had to work more shifts in recent months are affected by higher levels of occupational burnout, have less resilience, and experience more tension in their relationship with their children than before the emergence of the pandemic. In view of the persistence of the COVID-19 crisis in Iran and the world in the coming months, it is recommended that health administrators take effective measures such as increasing staff, increasing salaries, reducing work shifts, entertainment programs, and not working on holiday to reduce nurses' occupational burnout and enhance their physical and psychological well-being.

\section{Limitation}

One of the major limitations of the present study was the relatively low return rate of the questionnaires via e-mail, which could be due to the hectic work schedules of nurses in the crisis. Moreover, the variables addressed in the present study were measured over a 6 -month period - it is suggested that future studies assess occupational burnout, resilience, and parenting stress in nurses who care for COVID-19 patients in the coming months and years in other societies and larger samples in order to acquire a more accurate understanding of nurses' occupational burnout in this crisis. Health administrators and policy-makers can use these findings to develop more comprehensive plans for the current and future crises.

\section{Implications for nursing practice}

The nurse managers and policy makers of health organizations should examine and use the findings of this study in order to provide appropriate environments for nurses, and also to develop more comprehensive plans for the support of nurses during the current and future crises.

\section{Conclusions}

In the present study, the nurses who care for COVID-19 patients were found to be suffering from high levels of occupational burnout. Also, the results showed that nurses' occupational burnout correlates with their resilience, parenting stress, marital status, number of children, employment status, and gender: these variables predicted $61.32 \%$ of the subjects' occupational burnout variance.

\section{Acknowledgments}

The present article is extracted from a research project registered under the ethical code (umsha.ac.ir.1399.194) at Hamadan University of Medical Sciences, Hamadan, Iran. The authors would like to acknowledge Hamadan University of Medical Sciences for financially supporting this research. The researchers would like to express their gratitude to the authorities at Hamadan School of Nursing and Midwifery, the pre-hospital emergency care personnel who participated in the study, and all the other individuals who cooperated with them.

\section{Disclosure}

The authors declare no conflict of interest.

\section{References}

1. Abidin R, Flens JR, Austin WG. The Parenting Stress Index. In R. P. Archer (Ed.). Forensic uses of clinical assessment instruments. Lawrence Erlbaum Associates Publishers 2006; 297-328.

2. Ahmadi O, Azizkhani R, Basravi M. Correlation between workplace and occupational burnout syndrome in nurses. Adv Biomed Res 2014; 3: 1-4.

3. Amini F. The relationship between resiliency and burnout in nurses. J Res Develop Nurs Midwifery 2013; 10: 94-102.

4. Anthony LG, Anthony BJ, Glanville DN, et al. The relationships between parenting stress, parenting behaviour and preschoolers' social competence and behaviour problems in the classroom. Infant Child Develop Int I Res Pract 2005; 14: 133-154.

5. Baher A. The relationship between emotional intelligence and sense of self-efficacy and burnout among staff of Azad University of Tabriz. Training and Evaluation (Education) 2009; 2: 99-119.

6. Da Silva AA, Sanchez G, Barbosa NMS, de Oliveira MZ. Predictor variables for burnout among nursing professionals. Revista de psicología 2019a; 37: 319-348.

7. Da Silva Azevedo D, Martins Ferraz MM, Alencar Ferreira RDS, et al. Risk of burnout syndrome in mental health nurses. J Nurs UFPE/Revista de Enfermagem UFPE 2019b; 13: 978-986.

8. Edward KL, Hercelinskyj G. Burnout in the caring nurse: learning resilient behaviours. Br J Nurs 2007; 16: 240-242.

9. Greenberg N, Docherty M, Gnanapragasam S, Wessely S. Managing mental health challenges faced by healthcare workers during covid-19 pandemic. BMJ 2020; 368: m1211.

10. Lai CC, Shih TP, Ko WC, et al. Severe acute respiratory syndrome coronavirus 2 (SARS-CoV-2) and corona virus disease-2019 (COVID-19): the epidemic and the challenges. Int J Antimicrob Agents 2020a; 55: 105924.

11. Lai J, Ma S, Wang Y, et al. Factors associated with mental health outcomes among health care workers exposed to coronavirus disease 2019. JAMA Net Open 2020b; 3: e203976-e203976.

12. Lehmann M, Bruenahl CA, Löwe B, et al. Ebola and psychological stress of health care professionals. Emerg Infect Dis 2015; 21: 913-914. 
13. Liu S, Yang L, Zhang C, et al. Online mental health services in China during the COVID-19 outbreak. Lancet Psychiatry 2020; 7: e17-e18.

14. Maslach C, Jackson SE, Leiter MP, et al. Maslach burnout inventory. Vol. 21. Consulting Psychologists Press, Palo Alto, CA 1986.

15. Mohammadi F, Farjam M, Gholampour Y, et al. Caregivers' perception of the caring challenges in coronavirus crisis (COVID-19): a qualitative study. BMC Nurs 2021; 20: 1-9.

16. Mohammadi F, Farjam M, Gholampour Y, et al. Health professionals' perception of psychological safety in patients with coronavirus (COVID-19). Risk Manag Healthc Policy 2020; 13: 785-794

17. Nemati M, Ebrahimi B, Nemati F. Assessment of Iranian nurses' knowledge and anxiety toward COVID-19 during the current outbreak in Iran. Arch Clin Infect Dis 2020; 15 (COVID-19).

18. Novak M, Guest C. Application of a multidimensional caregiver burden inventory. Gerontologist 1989; 29: 798803.

19. Novel Coronavirus (2019). Situation report, 22. World Health Organization 2020.

20. Rafiee F, Oskouie F, Nikravesh M. Key factors in nurses' reaction to Burnout: A qualitative study. Razi J Med Sci 2007; 13: 83-94

21. Reitman D, Currier RO, Stickle TR. A critical evaluation of the Parenting Stress Index-Short Form (PSI-SF) in a head start population. J Clin Child Adolesc Psychol 2002; 31: 384-392.

22. Roy D, Tripathy S, Kar SK, et al. Study of knowledge, attitude, anxiety \& perceived mental healthcare need in Indian population during COVID-19 pandemic. Asian J Psychiatr 2020; 51: 102083.

23. Salahian A, Oreizi H, Babamiri M, Asgari A. The predictor factors of burnout syndrome in Isfahan nurses. Iran J Nurs Res 2012; 6: 23-31.

24. Sandeep K, Sandhyarani B, Rao ES, Sharma H. A study to assess knowledge regarding perceived causes of burnout syndrome among staff nurses at selected hospitals of Bareilly. Int J Adv Nurs Manag 2019; 7: 351-354.

25. Shafiezadeh A, Heravi-Karimooi M, Mirzaee A, et al. Psychometric characteristics of the Iranian Caregiver Burden Inventory $(\mathrm{CBI})$ in caregivers of elderly patients with $\mathrm{Al}$ zheimer. Health Qual Life Outcomes 2020; 18: 255.

26. Shirzadi P, Framarzi S, Ghasemi M, Shafiee M. Investigating validity and reliability of Parenting Stress Indexshort form among Fathers of normal child under 7 years old. Rooyesh-e-Ravanshenasi J 2015; 3: 91-110.

27. Sofology M, Efstratopoulou M, Dunn T. Predicting burnout syndrome in Greek mental health professionals. J Soc Serv Res 2019; 45: 142-149.

28. Stuijfzand S, Deforges C, Sandoz V, et al. Psychological impact of an epidemic/pandemic on the mental health of healthcare professionals: a rapid review. BMC Public Health 2020; 20: 1230 .

29. Torgheh M, Alipour A. Effect of humour on burnout and resiliency of nurses. J Holist Nurs Midwifery 2015; 25: 5764.

30. Van der Geest IM, Van den Heuvel-Eibrink MM, Passchier J, et al. Parenting stress as a mediator of parents' negative mood state and behavior problems in children with newly diagnosed cancer. Psychooncology 2014; 23: 758-765.

31. Velando-Soriano A, Ortega-Campos E, Gómez-Urquiza $J$, et al. Impact of social support in preventing burnout syndrome in nurses: A systematic review. Jpn J Nurs Sci 2020; 17: e12269.
32. Wang D, Hu B, Hu C, et al. Clinical characteristics of 138 hospitalized patients with 2019 novel coronavirus-infected pneumonia in Wuhan, China. JAMA 2020; 323: 10611069.

33. Wu Z, McGoogan JM. Characteristics of and important lessons from the coronavirus disease 2019 (COVID-19) outbreak in China: summary of a report of 72314 cases from the Chinese Center for Disease Control and Prevention. JAMA 2020; 323: 1239-1242.

34. Yeh CH, Chen ML, Li W, Chuang HL. The Chinese version of the parenting stress index: a psychometric study. Acta Paediatr 2001; 90: 1470-1477.

35. Zou G, Shen X, Tian X, et al. Correlates of psychological distress, burnout, and resilience among Chinese female nurses. Ind Health 2016; 54: 389-395. 